\title{
Mechanical loading-related changes in osteocyte sclerostin expression in mice are more closely associated with the subsequent osteogenic response than the peak strains engendered
}

\author{
A. Moustafa • T. Sugiyama • J. Prasad • G. Zaman • \\ T. S. Gross • L. E. Lanyon • J. S. Price
}

Received: 23 December 2010 / Accepted: 20 April 2011/Published online: 15 May 2011

(C) The Author(s) 2011. This article is published with open access at Springerlink.com

\begin{abstract}
Summary Osteocyte sclerostin is regulated by loading and disuse in mouse tibiae but is more closely related to subsequent local osteogenesis than the peak strains engendered.

Introduction The purpose of this study was to assess the relationship between loading-related change in osteocyte sclerostin expression, local strain magnitude, and local bone modeling/remodeling.

Methods The right tibiae of 19-week-old female C57BL/6 mice were subjected to non-invasive, dynamic axial loading and/or to sciatic neurectomy-induced disuse. The sclerostin status of osteocytes was evaluated immunohistochemically, changes in bone mass by micro-computed tomography, new bone formation by histomorphometry, and loading-induced strain by strain gauges and finite element analysis.

Results In cortical bone of the tibial shaft, loading engendered strains of similar peak magnitude proximally
\end{abstract}

A. Moustafa · T. Sugiyama · G. Zaman · L. E. Lanyon · J. S. Price Department of Veterinary Basic Sciences, The Royal Veterinary College, University of London,

London, UK

\section{A. Moustafa}

Department of Surgery, Faculty of Veterinary Medicine,

Kafr El-Sheikh University,

Kafr El-Sheikh, Egypt

T. Sugiyama $(\bowtie) \cdot$ L. E. Lanyon $\cdot$ J. S. Price

School of Veterinary Sciences, University of Bristol,

Langford House,

Langford, Bristol BS40 5DU, UK

e-mail: Toshihiro.Sugiyama@bristol.ac.uk

J. Prasad · T. S. Gross

Department of Orthopaedics and Sports Medicine,

University of Washington,

Seattle, WA, USA and distally. Proximally, sclerostin-positive osteocytes decreased and new bone formation increased. Distally, there was neither decrease in sclerostin-positive osteocytes nor increased osteogenesis. In trabecular bone of the proximal secondary spongiosa, loading decreased sclerostin-positive osteocytes and increased bone volume. Neither occurred in the primary spongiosa. Disuse increased sclerostin-positive osteocytes and decreased bone volume at all four sites. Loading reversed this sclerostin upregulation to a level below baseline in the proximal cortex and secondary spongiosa.

Conclusion Loading-related sclerostin downregulation in osteocytes of the mouse tibia is associated preferentially with regions where new bone formation is stimulated rather than where high peak strains are engendered. The mechanisms involved remain unclear, but could relate to peak surface strains not accurately reflecting the strain-related osteogenic stimulus or that sclerostin regulation occurs after sufficient signal processing to distinguish between local osteogenic and non-osteogenic responses.

Keywords Disuse · Finite element analysis · Mechanical loading $\cdot$ Mechanical strain $\cdot$ Osteocyte $\cdot$ Sclerostin

\section{Introduction}

Habitual loading has a profound influence on bone mass and architecture mediated by the effects on resident bone cells of the dynamic changes in local mechanical strain engendered [1]. In general, high or unusually distributed strains stimulate increases in new bone formation, and thus a more robust structure, whereas low strains, as seen in disuse, are associated with bone resorption and a weaker 
one. The high incidence of fragility fractures in postmenopausal women suggests a failure of this natural regulatory process since continued functional loading is accompanied by loss of bone tissue and an increase in bone fragility.

The recent identification of sclerostin as a molecule preferentially secreted by osteocytes [2-4] that appears to be regulated by bone's mechanical environment [5-11] has attracted considerable interest, particularly because sclerostin-neutralizing antibodies engender a prolonged osteogenic response $[12,13]$. The mechanism by which mechanical strain could exert its effect through sclerostin is envisaged to be by inhibition of the Wnt-signaling pathway [14-16]. Exposure to mechanical strain, by suppressing sclerostin production, would increase the osteogenic effect of the Wnt pathway. This is consistent with the situation in genetically modified mice where deficiency in functional sclerostin expression is linked to increased bone formation and bone mass $[8,17]$, as it is in humans with sclerosteosis $[18,19]$ or van Buchem disease [20, 21]. Polymorphic variation in the SOST locus coding for sclerostin has also been shown to contribute to the genetic regulation of areal bone mineral density and fracture risk [22]. In patients with hip fracture, sclerostin-positive osteocyte staining appears to increase more sharply with osteonal maturation than in non-fracture controls [23].

In the present study, we assessed whether sclerostin regulation in osteocytes is directly linked to local changes in the magnitude of peak strains engendered by mechanical loading. To do this, we used the mouse unilateral tibia axial loading model [24, 25] and measured loading-related changes in osteocyte sclerostin expression in both cortical and trabecular bone. These changes were then compared to the local strains engendered and the subsequent local bone modeling/remodeling. Our data suggest that loading-related changes in osteocyte sclerostin expression are more closely associated with the subsequent osteogenic response than the peak strains engendered.

\section{Materials and methods}

\section{Animals}

Virgin, female C57BL/6 mice at 7-8 weeks of age were purchased from Charles River Laboratories, Inc. (Margate, UK) and group-housed in sterilized polypropylene cages with free access to water and a maintenance diet containing $0.73 \%$ calcium, $0.52 \%$ phosphorus, and $3.5 \mathrm{IU} / \mathrm{g}$ vitamin D (RM1; Special Diet Services Ltd., Witham, UK) in a 12h light/dark cycle, with room temperature at $21^{\circ} \mathrm{C} \pm 2^{\circ} \mathrm{C}$. The mice were used for experiments when almost skeletally mature at 19 weeks of age. All procedures complied with the UK Animals (Scientific Procedures) Act 1986 and were reviewed and approved by the ethics committee of the Royal Veterinary College (London, UK).

In vivo external mechanical loading

The apparatus and protocol for axial loading of the mouse tibia have been reported previously [24-26]. Noninvasive, dynamic loads $[0.1 \mathrm{~s}$ trapezoidal-shaped pulse (period $0.025 \mathrm{~s}$ loading, $0.05 \mathrm{~s}$ hold, and $0.025 \mathrm{~s}$ unloading); $10 \mathrm{~s}$ rest time between each pulse; 40 cycles/ day] were applied between the right flexed knee and ankle under isoflurane-induced anesthesia (approximately $7 \mathrm{~min} /$ day). This rest time enhances the osteogenic potential of loading [27]. The flexed joints are positioned in concave cups; the upper cup, into which the knee is positioned, is attached to the actuator arm of a servo-hydraulic loading machine (Model HC10; Zwick Testing Machines Ltd., Leominster, UK) and the lower cup to a dynamic load cell. The servo-hydraulic mechanism of the loading machine operates to apply controlled dynamic compressive loads axially to the tibia. The left non-loaded tibia was used as an internal control, as has previously been validated in the present model [25] and confirmed by others in the rat ulna axial loading model [28]. Normal activity within the cages was allowed between loading periods. In the present study, a peak load of $13.5 \mathrm{~N}$ was selected since this has previously been shown to induce significant bone gain through an increase in bone formation at both cortical and trabecular sites [7, 25].

\section{Assessment of loading-induced strain}

Single element strain gauges were attached ex vivo, in a longitudinal orientation, to the proximal lateral tibial shaft of similar 19-week-old female C57BL/6 mice. These showed that a peak load of $13.5 \mathrm{~N}$ engendered a peak longitudinal strain of approximately $1,800 \mu \varepsilon$ in that region. Since the mouse tibia is not large enough to permit attachment of multiple gauges, the predictions of the normal strain distribution throughout the bone induced by loading were extended to full bone normal strain characterizations using finite element (FE) analysis. A voxel-based FE model (voxel size, $40 \mu \mathrm{m}$ ) was constructed by processing the micro-computed tomography $(\mu \mathrm{CT})$ images using a computer program developed in house in the Department of Orthopaedics and Sports Medicine, University of Washington [29]. The bone material properties were assumed to be homogeneous, linear, and isotropic (Young's modulus, $17 \mathrm{GPa}$; Poisson's ration, 0.3) in order to approximately match the above strain gauge reading. FE analysis was implemented, as previously reported [29-31], at the proximal and distal sites in cortical bone of the tibiae (37\% and $75 \%$ of the bone's length from its proximal end, 
respectively) and trabecular bone sites $0.01-0.05 \mathrm{~mm}$ (mainly primary spongiosa) and $0.05-1.00 \mathrm{~mm}$ (secondary spongiosa) distal to the growth plate of the proximal tibiae. For the analysis of cortical bone, the transverse section of the bone was divided into regions parallel to the neutral axis equating to different magnitudes of strain in tension or corresponding strains in compression.

\section{"Loading" experiments}

Where loading was to be related to sclerostin regulation, the right tibiae of mice $(n=6)$ were subjected to loading on two consecutive days. Left non-loaded control and right loaded tibiae were collected $24 \mathrm{~h}$ after the second period of loading. These bones were dissected free of soft tissue, fixed in $10 \%$ buffered formalin, and decalcified in formic acid (Immuncal; Decal Chemical Corp. Tallman, NY, USA) for immunohistochemistry.

Where loading was to be related to changes in bone modeling/remodeling, loading was applied to the right tibiae of an additional six mice on three alternate days per week for 2 weeks (days 1, 3, 5, 8, 10, and 12). High doses of calcein (50 mg/kg; Sigma Chemical Co., St. Louis, MO, USA) and alizarin $(50 \mathrm{mg} / \mathrm{kg}$; Sigma Chemical Co.) were injected intraperitoneally on the first and last days of loading (days 1 and 12), respectively. At 21 weeks of age (day 15), the mice were euthanized and their left and right tibiae were collected and fixed in $70 \%$ ethanol for $\mu \mathrm{CT}$ analysis and histomorphometry.

\section{"Disuse/loading" experiments}

Where sclerostin regulation in the tibiae was to be assessed in the situation of disuse, mice were subjected to unilateral sciatic neurectomy or sham sciatic neurectomy (day 1). Sciatic neurectomy was performed by resecting a 3- to 4-mm segment of the right sciatic nerve posterior to the hip joint under isoflurane-induced anesthesia. Eight mice with right sciatic neurectomy were randomly divided into two groups; the right tibiae of one group $(n=4)$ received loading on days 3 and 4 , while the other group $(n=4)$ received no artificial loading. Since surgical intervention could potentially increase sclerostin expression [32, 33], an additional six mice received right sham sciatic neurectomy without artificial loading to act as controls. Both the left and right tibiae of all the mice were collected on day 5 ( $24 \mathrm{~h}$ after the second period of loading), dissected of soft tissue, fixed in $4 \%$ paraformaldehyde, and decalcified in 14\% EDTA for immunohistochemistry.

To assess the site-specific degree of bone loss after sciatic neurectomy, six mice received right sciatic neurectomy and were sacrificed 3 weeks later (at 22 weeks of age) without having received any artificial loading. Their left and right tibiae were collected and fixed in $70 \%$ ethanol for $\mu \mathrm{CT}$ analysis.
Sclerostin immunohistochemistry

Sclerostin was immunolocalized at (1) proximal and distal sites in the cortical bone of the tibiae $(37 \%$ and $75 \%$ of the bone's length from its proximal end, respectively) and (2) primary and secondary spongiosa in trabecular bone of the proximal tibiae, in decalcified, wax-embedded $8-\mu \mathrm{m}$ transverse sections using an indirect immunoperoxidase method as reported previously [7]. Goat polyclonal anti-mouse sclerostin $(0.2 \mathrm{mg} / \mathrm{ml}$; R\&D Systems, Abingdon, UK) and biotinylated rabbit anti-goat $(0.013 \mathrm{mg} / \mathrm{ml}$; Dako, Ely, UK) were used as the primary and secondary antibodies, respectively. All antibodies were diluted in $10 \%$ rabbit serum (Sigma Chemical Co.) in calcium and magnesiumfree phosphate-buffered saline (Gibco, Paisley, UK). The same concentration of goat $\operatorname{IgG}$ was substituted for the primary antibody to provide a negative control. The detection of sclerostin was achieved using a vector $\mathrm{ABC}$ kit (Vector Laboratories, Burlingame, CA, USA) with diaminobenzidine as a substrate. The immunolabeled sections were photographed using a Leica DMR microscope (Leica Microsystems, Heidelberg, Germany). The numbers of sclerostin-positive and total osteocytes were counted, and the changes in osteocyte sclerostin expression by loading and/or sciatic neurectomy-related disuse were calculated as percentage changes compared to the control tibia for each animal [(right loaded-left control) $\times 100 /$ left control] at the proximal and distal sites of cortical bone and in the primary and secondary spongiosa of trabecular bone. At these two cortical sites, the percentages of sclerostin-positive osteocytes were also measured at regions corresponding to different levels of strain determined by FE analysis.

\section{$\mu \mathrm{CT}$ analysis}

All tibiae analysed by $\mu \mathrm{CT}$ (SkyScan 1172; SkyScan, Kontich, Belgium) were scanned with a pixel size of $5 \mu \mathrm{m}$. Images of the whole bones were reconstructed with SkyScan software and three-dimensional structural analyses were performed for (1) $0.5-\mathrm{mm}$ long sections at the proximal and distal sites in cortical bone of the tibiae (37\% and $75 \%$ of the bone's length from its proximal end, respectively) and (2) trabecular bone sites $0.01-$ $0.05 \mathrm{~mm}$ (mainly primary spongiosa) and $0.05-1.00 \mathrm{~mm}$ (secondary spongiosa) distal to the growth plate of the proximal tibiae. The parameters evaluated included cortical bone volume and trabecular bone volume/tissue volume $(\mathrm{BV} / \mathrm{TV})$.

\section{Histomorphometry}

After scanning by $\mu \mathrm{CT}$, the bones were dehydrated and embedded in methyl methacrylate as previously described 
[25]. Transverse segments were obtained by cutting with an annular diamond saw. Images of calcein- and alizarinlabeled bone sections were visualized using an argon $488 \mathrm{~nm}$ laser and a HeNe $543 \mathrm{~nm}$ laser, respectively, on a confocal laser scanning microscope (LSM 510; Carl Zeiss MicroImaging $\mathrm{GmbH}$, Jena, Germany) at similar cortical regions as the FE analysis, sclerostin immunohistochemistry, and $\mu \mathrm{CT}$ analysis. Using ImageJ software (version 1.42; http://rsbweb.nih.gov/ij/), periosteal and endosteal labels and inter-label bone areas were measured as loading-related newly formed bone area at regions corresponding to different levels of strain determined by $\mathrm{FE}$ analysis and were normalized to cortical bone area in each region.

\section{Statistical analysis}

All data are shown as the means \pm SE. Statistical analysis was performed by one-way ANOVA followed by a post hoc Dunnett T3 test or paired $t$ test using SPSS for Windows (version 17.0; SPSS Inc., Chicago, USA) and $p<0.05$ was considered statistically significant.
Fig. 1 Relationship between mechanical loading-related changes in osteocyte sclerostin expression and magnitudes of local strain engendered vs. subsequent osteogenesis in cortical bone. a Transverse loadinginduced strain distribution by $\mathrm{FE}$ analysis at the proximal and distal sites $(37 \%$ and $75 \%$ of the bone's length from its proximal end, respectively) of the tibia. Bone area was divided into five regions parallel to the neutral axis (region 0 ) corresponding to different magnitudes of strain in tension (region $+\mathrm{I}$ ) or compression (regions - I to - III). b Representative transverse fluorochrome-labeled images at the proximal and distal sites of the left control and right loaded tibiae. Green: calcein label injected on the first day of loading. Red: alizarin label injected on the last day of loading. c Loading-related increase in newly formed bone area and decrease in sclerostinpositive osteocytes in each of the five regions (corresponding to different strain magnitudes) at the proximal and distal sites. Bars represent the means $\pm \mathrm{SE}$ $(n=6) . * p<0.05$ vs. region 0 a

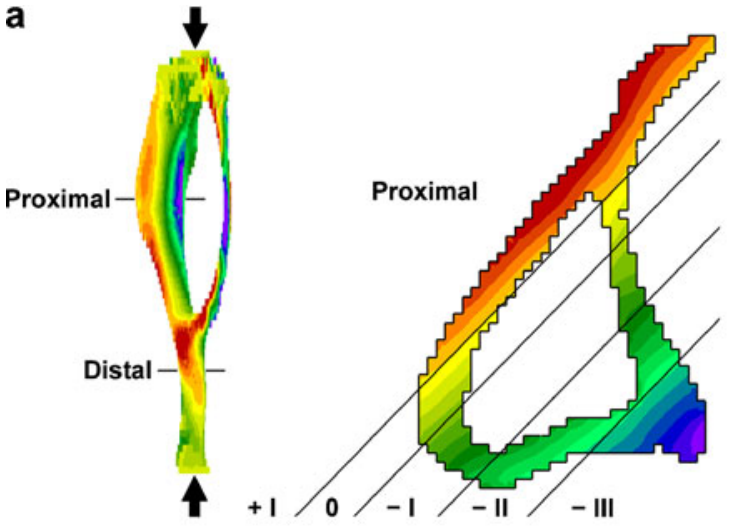

b

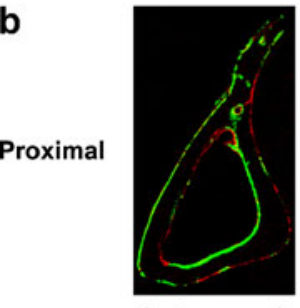

Left control

C

Loading-related increase in newly-formed bone area $\left(\%\left[\times 10^{-2}\right]\right)$

Loading-related decrease in sclerostin-positive osteocytes

(\%)

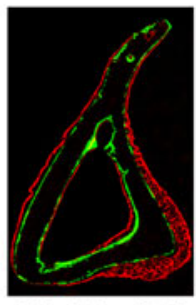

Right loaded

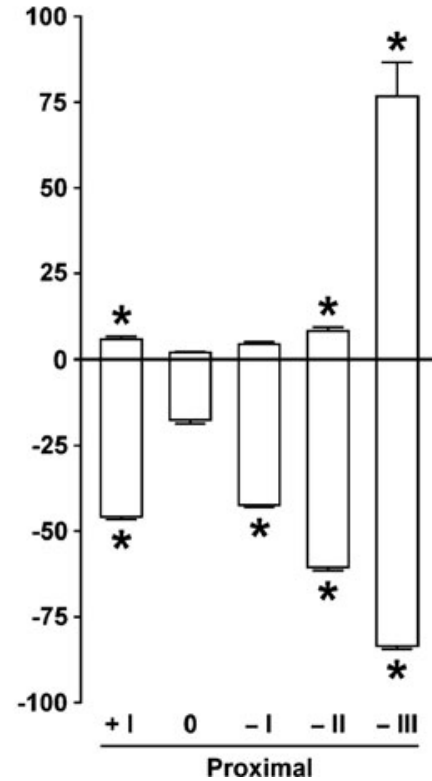

Distal
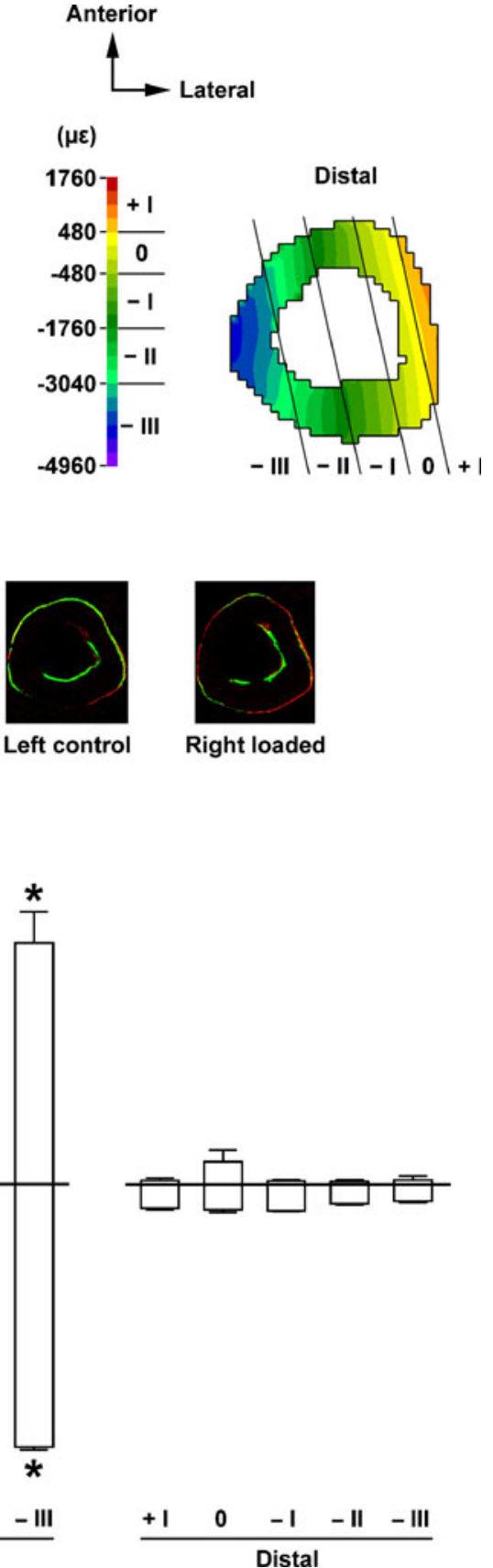


\section{Results}

Effects of mechanical loading

Figure 1a shows images of the loading-induced strain distribution as determined by FE analysis. Transverse sections of the tibia at the proximal and distal cortical sites are shown with the strain distribution across the section divided into five regions parallel to the neutral axis according to strain magnitude [region $+\mathrm{I}(+480$ to $+1,760 \mu \varepsilon)$, region 0 $(-480$ to $+480 \mu \varepsilon)$, region $-\mathrm{I}(-480$ to $-1,760 \mu \varepsilon)$, region - II $(-1,760$ to $-3,040 \mu \varepsilon)$, and region - III $(-3,040$ to $-4,960 \mu \varepsilon)]$. In region 0 of the proximal section, there was no difference in new bone formation between left control and right loaded tibiae. In regions $+\mathrm{I},-\mathrm{II}$, and $-\mathrm{III}$, there were significant loading-related increases in new bone formation, reaching a 75 -fold increase in region -III. The magnitude of loading-related decrease in the percentage of sclerostin-positive osteocytes mirrored the amount of loading-related osteogenesis (Fig. 1). In contrast, there was no significant effect of loading on either new bone formation or the percentage of sclerostin-positive osteocytes in any region of the distal sections.

In trabecular bone of the proximal tibia, FE analysis suggested that loading-induced strain levels were lower in the primary spongiosa than in the secondary spongiosa (Fig. 2a). In the secondary spongiosa but not in the primary spongiosa, there was a loading-related decrease in the percentage of sclerostin-positive osteocytes (Fig. 2b) and a loading-related increase in trabecular $\mathrm{BV} / \mathrm{TV}$ (Fig. 2c).

Effects of sciatic neurectomy-induced disuse

Sciatic neurectomy was associated with a higher percentage of sclerostin-positive osteocytes in cortical bone at both the proximal and distal sites of the tibial shaft (Fig. 3a, b) and in trabecular bone of both the primary and secondary spongiosa of the proximal tibia (Fig. 4a, b). In the cortical bone, it was notable that it was not only the osteocyte cell bodies but also the canalicular network which was strongly immunostained for sclerostin shortly after sciatic neurectomy (Fig. 3a). In contrast, sham sciatic neurectomy had no effects on osteocyte sclerostin expression in either cortical bone (proximal; control $60 \% \pm 1 \%$ vs. sham $58 \% \pm 1 \%$, distal; control $64 \%$ $\pm 1 \%$ vs. sham $61 \% \pm 1 \%$ ) or trabecular bone (primary; control $76 \pm 2 \%$ vs. sham $72 \pm 2 \%$, secondary; control $72 \% \pm 4 \%$ vs. sham $74 \% \pm 1 \%$ ). Cortical bone volume at the proximal and distal sites (Fig. 3c) and trabecular BV/ $\mathrm{TV}$ in the primary and secondary spongiosa (Fig. 4c) were all significantly decreased 3 weeks after sciatic neurectomy. a
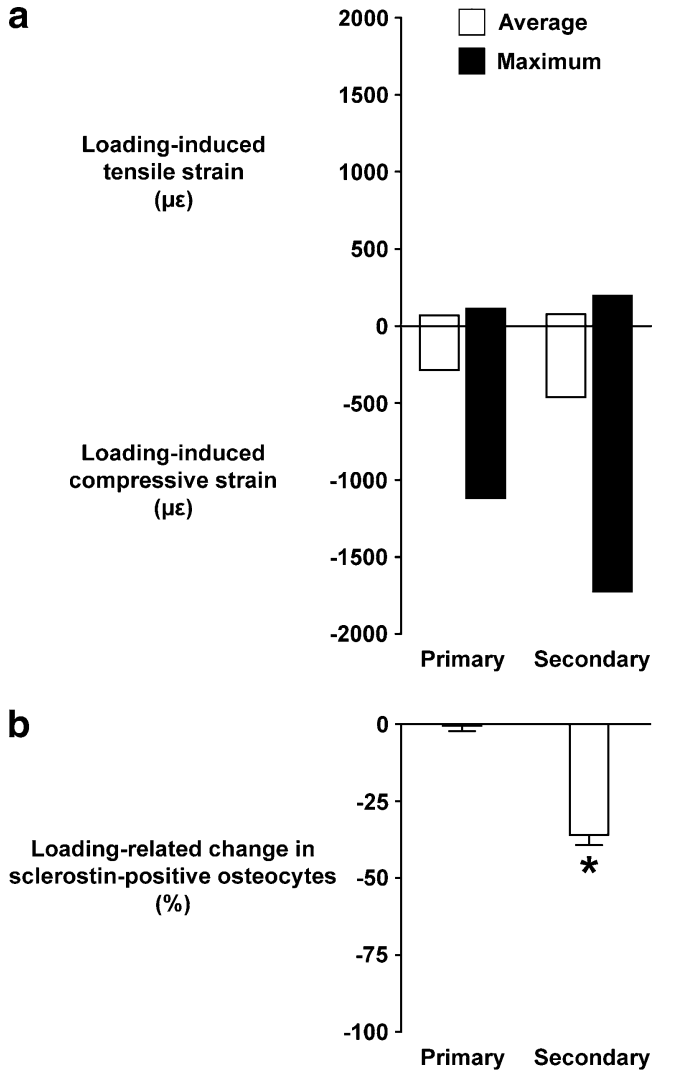

C

Loading-related change in trabecular BV/TV

(\%)

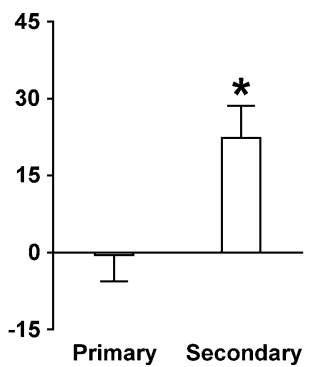

Fig. 2 Relationship between mechanical loading-related changes in osteocyte sclerostin expression and magnitudes of local strain engendered vs. subsequent changes in bone mass in trabecular bone. a Loading-induced tensile and compressive strain magnitudes, predicted by FE analysis, in the primary and secondary spongiosa of the proximal tibia. b Loading-related change in sclerostin-positive osteocytes in the primary and secondary spongiosa of the proximal tibia. c Loading-related change in trabecular BV/TV in the primary and secondary spongiosa of the proximal tibia. Bars represent the means \pm SE $(n=6) .{ }^{*} p<0.05$

Loading reversed the sciatic neurectomy-induced increases in the percentage of sclerostin-positive osteocytes in the cortical bone of both the proximal and distal sites (Fig. 3a, b) and in the trabecular bone of both the primary and secondary spongiosa (Fig. 4a, b). However, loading reduced the percentage of sclerostin-positive osteocytes to a level significantly lower than that in controls only in the proximal cortical region and the secondary spongiosa. 
Fig. 3 Disuse-related changes in osteocyte sclerostin expression and bone mass in cortical bone. a Sclerostin immunolocalization in transverse sections at the proximal and distal sites (37\% and $75 \%$ of the bone's length from its proximal end, respectively) of the left control, right immobilized, and right immobilized then loaded tibiae. $B a r=50 \mu \mathrm{m}$. b The percentage of sclerostin-positive osteocytes at the proximal and distal sites of the left control, right immobilized, and right immobilized then loaded tibiae. Bars represent the means $\pm \operatorname{SE}(n=4)$. c Cortical bone volume at the proximal and distal sites of the left control and right immobilized tibiae. Bars represent the means \pm SE $(n=6) .{ }^{*} p<0.05$. $C$ control, $S N$ sciatic neurectomy, $L$ loading

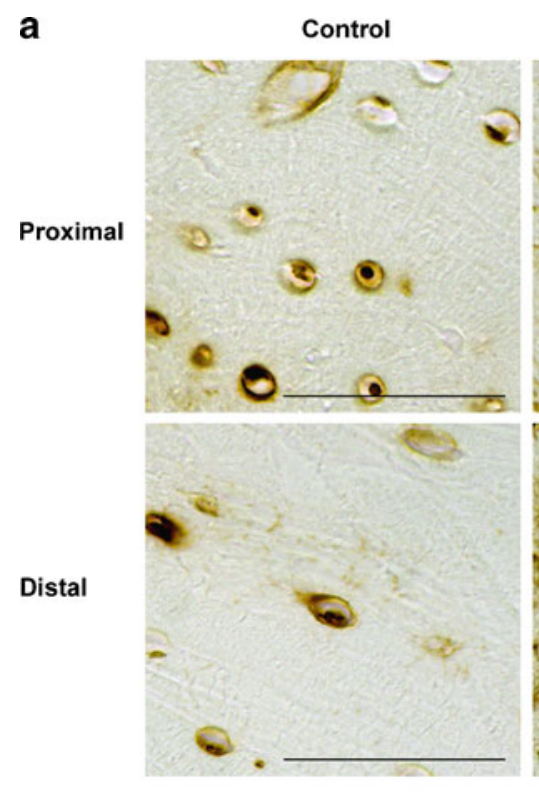

a

b

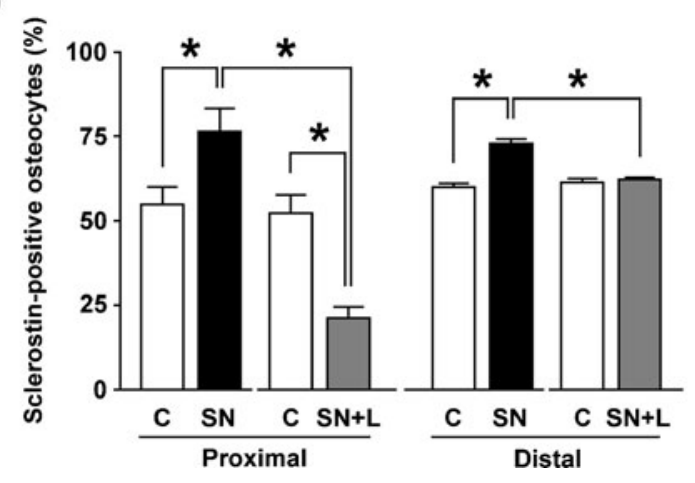

Siatic neurectomy

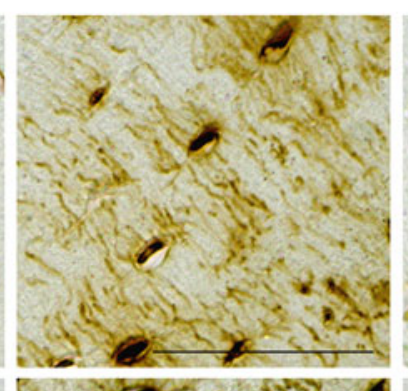

Sciatic neurectomy + loading
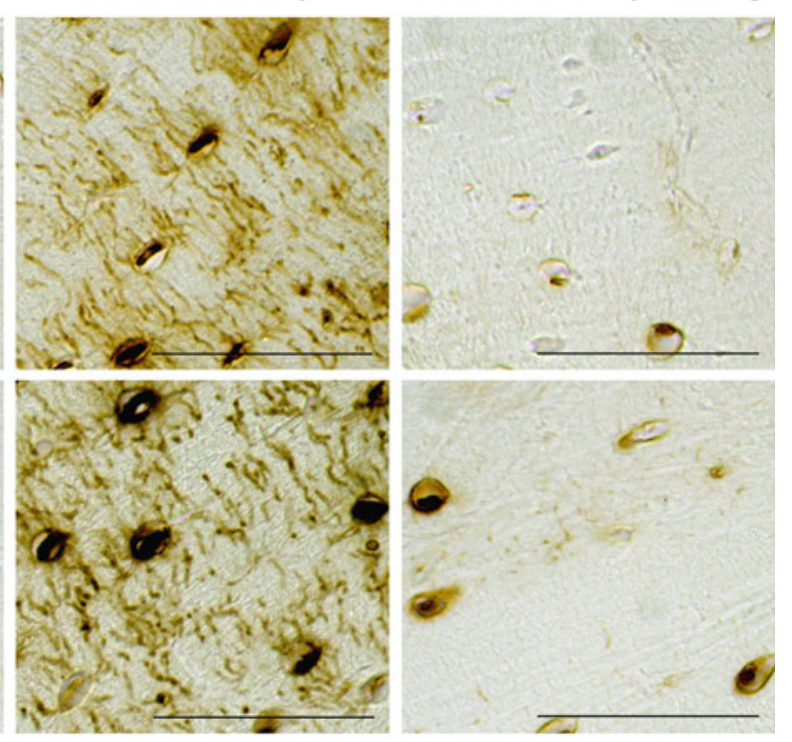

C

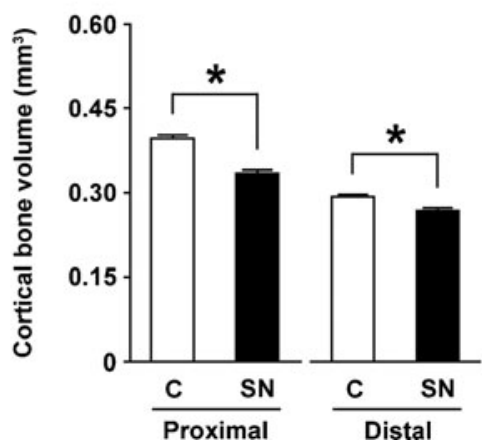

\section{Discussion}

In the present study, we used the mouse unilateral tibia axial loading model $[24,25]$ to assess the effects of loading on both cortical and trabecular bone compartments in vivo. In cortical bone, short periods of dynamic loading, in addition to that engendered by habitual physical activity, were associated with decreased osteocyte sclerostin staining and increased bone formation and bone volume at the proximal but not the distal site. In contrast, reduced loading due to sciatic neurectomy resulted in an increase in the percentage of sclerostin-positive osteocytes and decreased bone volume at both sites. In trabecular bone, exposure to the same artificial loading regimen induced a decrease in osteocyte sclerostin staining and an increase in bone volume in the secondary but not the primary spongiosa. Sciatic neurectomy-related disuse caused an increase in osteocyte sclerostin staining and a decrease in bone volume in both primary and secondary spongiosa. The effects of sciatic neurectomy-related disuse on both cortical and trabecular bone were reversed by artificial loading, with a significant reduction in sclerostin expression, to below that seen in controls, at the proximal site and secondary spongiosa, respectively.

The analysis of loading-related strain levels, areas of new bone formed, and changes in the sclerostin status of osteocytes in cortical bone confirmed that sclerostin downregulation by loading was not uniform throughout the bone, and revealed that this was less closely associated with the magnitude of peak strain engendered than with the degree of subsequent local new bone formation. In the proximal cortical region, loading-related suppression of osteocyte sclerostin expression was linked to the area of loadingrelated newly formed bone. Loading-induced strain magnitude is frequently associated with subsequent bone formation, and at the proximal site, the strain distribution map we present, which is similar to that reported by others [30], was also related to the area of loading-related newly formed bone. These data are consistent with the results reported previously [6]. However, this association did not hold at the distal site, where similar peak magnitudes of strain were unaccompanied by subsequent new bone formation and 
Fig. 4 Disuse-related changes in osteocyte sclerostin expression and bone mass in trabecular bone. a Sclerostin immunolocalization in longitudinal sections in the primary and secondary spongiosa of the left control, right immobilized, and right immobilized then loaded tibiae. Bar $=50 \mu \mathrm{m}$. b The percentage of sclerostin-positive osteocytes in the primary and secondary spongiosa of the left control, right immobilized, and right immobilized then loaded tibiae. Bars represent the means \pm SE ( $n$ $=4)$. $\mathbf{c}$ Trabecular BV/TV in the primary and secondary spongiosa of the left control and right immobilized tibiae. Bars represent the means $\pm \operatorname{SE}(n=6) .{ }^{*} p<$ 0.05 . $C$ control, $S N$ sciatic neurectomy, $L$ loading a

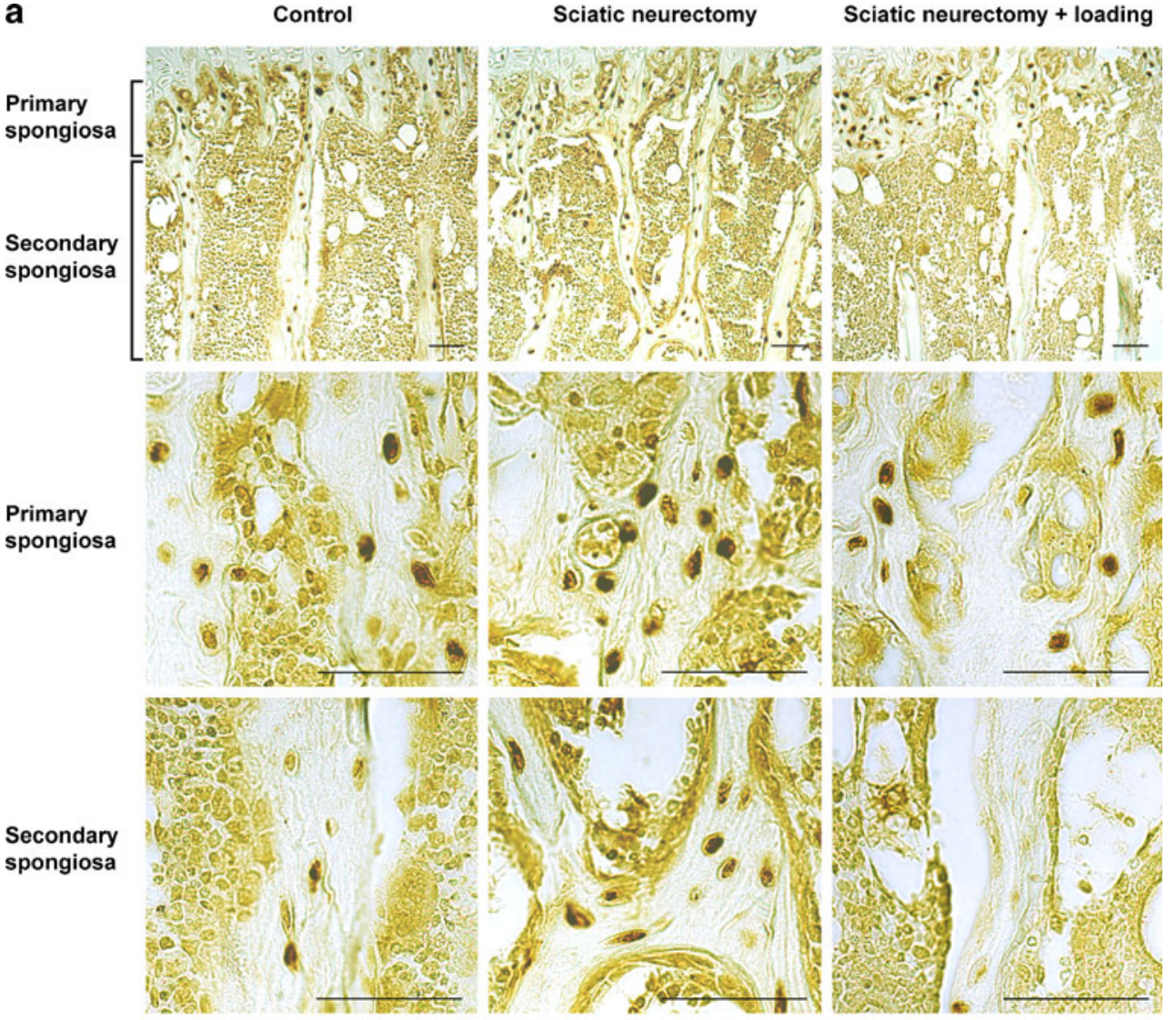

b

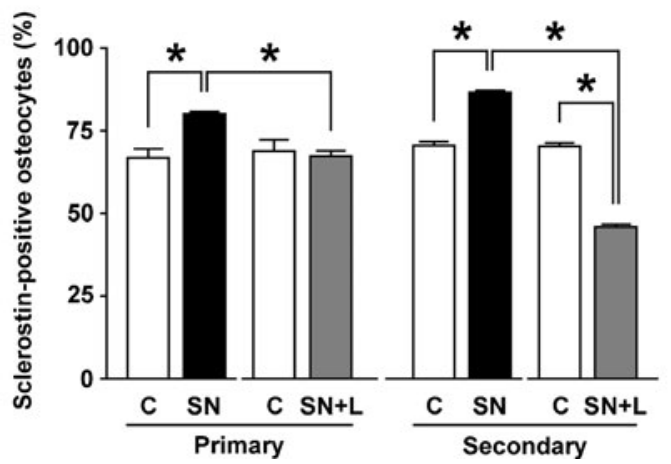

C

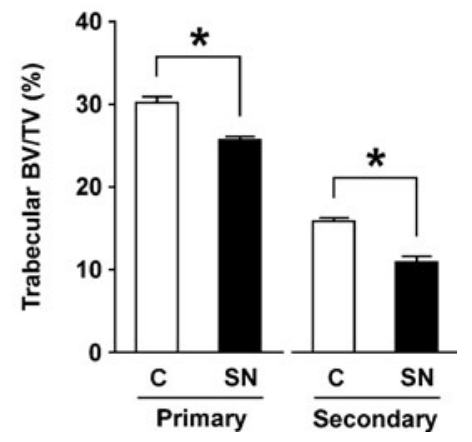

were not associated with change in the levels of osteocyte sclerostin.

The finding that axial loading stimulates peak strain magnitude-related increases in bone formation in some regions, but not others, is compatible with previously reported findings in the ulna [34]. One possible explanation for such variability in response at different regions within a single bone is that the osteogenic stimulus is more closely related to components of the strain regimen such as strain gradients than to peak surface strain magnitude [35]. As shown in Fig. 1a, the longitudinal curvature of the tibia's proximal region deviates from the axis of loading while the proximal region is better aligned to that axis. Thus, strain gradients at the distal site would be lower than the proximal site due to less bending. It must always also be born in mind that the bulk strain estimates, derived from strain gauges and predicted by FE analysis, do not necessarily reflect the actual strains in the matrix around osteocyte lacunae. These strains are heterogeneous and may be much higher than the applied macroscopic strains [36, 37]. However, we have no reason to believe from the immunocytochemistry that, at the level of the osteocyte, there was any heterogeneity with a distribution which could account for differences in the regional response.

There are a number of possible explanations for why there is a lack of consistent association between surface bone strain, sclerostin downregulation, and local new bone formation. One is that osteocytes respond directly in their 
sclerostin regulation to aspects of the strain regimen with different osteogenic potential (such as strain gradients and possibly their derivative fluid flow [35]) that are not reflected in the surface strain recordings. More likely in our view is that osteocytes respond directly to their local strain environment, including strain gradients, etc., but that they regulate their sclerostin production after sufficient processing of this initial strain-related stimulus to distinguish between osteogenic and non-osteogenic responses.

Differential regulation of sclerostin and osteogenesis in the primary and secondary spongiosa has also previously been reported following intermittent parathyroid hormone (PTH) treatment. Similarly to the effect of loading, intermittent PTH resulted in greater suppression of sclerostin [38] and increased bone gain [39] in the secondary than in the primary spongiosa. This would support the hypothesis that in trabecular as well as cortical bone, loadingrelated changes in osteocyte sclerostin suppression are associated with the osteogenic response to loading. If this were the case, it suggests that osteocyte sclerostin suppression is a feature of the early (re)modeling control stimulus resulting from interactions within bone cells between a number of pathways whose activity can be altered by mechanical strain. The downregulation of sclerostin would then be indicative of an early osteogenic response to strain rather than a consequence of strain itself. At present, the specificity of sclerostin's response to strain and association with osteogenesis remain to be determined.

Sciatic neurectomy resulted in a non-site-specific increase in osteocyte sclerostin expression in both cortical and trabecular bone. This upregulation was not observed following sham sciatic neurectomy. The uniform increase in sclerostin expression with sciatic neurectomy-induced disuse contrasts with the regional effects seen with loading, probably because the effect of disuse induced by sciatic neurectomy is a uniform reduction in mechanical strain [40]. Our data, in 19week-old female mice, are not perfectly consistent with those of others using tail suspension, in 6-week-old male mice, where unloading was associated with an increase in the expression of the sost gene but not the sclerostin protein [6]. Potential reasons for this discrepancy include the possibility that tail suspension permits continued muscle activity which, even in the absence of ground reaction forces, may engender significant changes in bone strain. Nevertheless, mice lacking the sost gene showed resistance to bone loss induced by tail suspension in both cortical and trabecular regions [8].

The relevance of the present short-term experiment in mice to the human condition must take into account a number of differences in the two situations including the pattern of their normal bone modeling and remodeling. However, the implication of this study for our understand- ing of the potential role of sclerostin in loading and disuserelated control of bone (re)modeling is probably transferable. Indeed, in agreement with our experimental data, immobilization-induced bone loss in stroke patients is associated with a state of "hypersclerostinemia" [41]. The circulating sclerostin levels in humans negatively correlate with the circulating PTH levels [42] and osteocytic Sost suppression is likely to mediate the effects of intermittent PTH [43, 44] which synergistically enhances loadingrelated osteogenesis in mice [45]. Sclerostin-neutralizing drugs $[12,13]$ therefore have great potential to provide an effective anabolic treatment for the prevention of fragility fractures in humans.

In conclusion, the present data from both cortical and cancellous bone in adult female mice suggest a substantial regulation of osteocyte sclerostin production by bone's mechanical environment. Exposure to loading is generally associated with downregulation and disuse with upregulation. However, osteocyte sclerostin status appears to be less closely related to the magnitude of local loading-related strain, as determined by surface-bonded strain gauges and by $\mathrm{FE}$ analysis, than to the subsequent increase in new bone formation. Further studies are required to elucidate the mechanistic association between changes in osteocytic sclerostin expression and local new bone formation. At present, we are unable to determine whether osteocytes respond directly in their sclerostin regulation to aspects of the strain regimen with different osteogenic potential that are not reflected in the surface strain recordings, or whether they respond directly to local strains but regulate their sclerostin production after sufficient processing of this initial strain-related stimulus to distinguish between osteogenic and non-osteogenic responses.

Acknowledgments This work was supported by the Wellcome Trust (to L. E. Lanyon and J. S. Price) and NIH AR60304 (to T. S. Gross). A. Moustafa is supported by the Egyptian Ministry of Higher Education.

Conflicts of interest None.

Open Access This article is distributed under the terms of the Creative Commons Attribution Noncommercial License which permits any noncommercial use, distribution, and reproduction in any medium, provided the original author(s) and source are credited.

\section{References}

1. Price JS, Sugiyama T, Galea GL, Meakin LB, Sunters A, Lanyon LE (2011) Role of endocrine and paracrine factors in the adaptation of bone to mechanical loading. Curr Osteoporos Rep 9:76-82

2. Winkler DG, Sutherland MK, Geoghegan JC, Yu C, Hayes T, Skonier JE, Shpektor D, Jonas M, Kovacevich BR, StaehlingHampton K, Appleby M, Brunkow ME, Latham JA (2003) 
Osteocyte control of bone formation via sclerostin, a novel BMP antagonist. EMBO J 22:6267-6276

3. van Bezooijen RL, Roelen BA, Visser A, van der Wee-Pals L, de Wilt E, Karperien M, Hamersma H, Papapoulos SE, ten Dijke P, Lowik CW (2004) Sclerostin is an osteocyte-expressed negative regulator of bone formation, but not a classical BMP antagonist. J Exp Med 199:805-814

4. Poole KE, van Bezooijen RL, Loveridge N, Hamersma H, Papapoulos SE, Lowik CW, Reeve J (2005) Sclerostin is a delayed secreted product of osteocytes that inhibits bone formation. FASEB J 19:1842-1844

5. Tatsumi S, Ishii K, Amizuka N, Li M, Kobayashi T, Kohno K, Ito M, Takeshita S, Ikeda K (2007) Targeted ablation of osteocytes induces osteoporosis with defective mechanotransduction. Cell Metab 5:464-475

6. Robling AG, Niziolek PJ, Baldridge LA, Condon KW, Allen MR, Alam I, Mantila SM, Gluhak-Heinrich J, Bellido TM, Harris SE, Turner CH (2008) Mechanical stimulation of bone in vivo reduces osteocyte expression of Sost/sclerostin. J Biol Chem 283:58665875

7. Moustafa A, Sugiyama T, Saxon LK, Zaman G, Sunters A, Armstrong VJ, Javaheri B, Lanyon LE, Price JS (2009) The mouse fibula as a suitable bone for the study of functional adaptation to mechanical loading. Bone 44:930-935

8. Lin C, Jiang X, Dai Z, Guo X, Weng T, Wang J, Li Y, Feng G, Gao X, He L (2009) Sclerostin mediates bone response to mechanical unloading through antagonizing Wnt/beta-catenin signaling. J Bone Miner Res 24:1651-1661

9. Bonnet N, Standley KN, Bianchi EN, Stadelmann V, Foti M, Conway SJ, Ferrari SL (2009) The matricellular protein periostin is required for sost inhibition and the anabolic response to mechanical loading and physical activity. J Biol Chem 284:35939-35950

10. Callewaert F, Bakker A, Schrooten J, Van Meerbeek B, Verhoeven G, Boonen S, Vanderschueren D (2010) Androgen receptor disruption increases the osteogenic response to mechanical loading in male mice. J Bone Miner Res 25:124-131

11. Zaman G, Saxon LK, Sunters A, Hilton H, Underhill P, Williams D, Price JS, Lanyon LE (2010) Loading-related regulation of gene expression in bone in the contexts of estrogen deficiency, lack of estrogen receptor alpha and disuse. Bone 46:628-642

12. Ominsky MS, Vlasseros F, Jolette J, Smith SY, Stouch B, Doellgast G, Gong J, Gao Y, Cao J, Graham K, Tipton B, Cai J, Deshpande R, Zhou L, Hale MD, Lightwood DJ, Henry AJ, Popplewell AG, Moore AR, Robinson MK, Lacey DL, Simonet WS, Paszty C (2010) Two doses of sclerostin antibody in cynomolgus monkeys increases bone formation, bone mineral density, and bone strength. J Bone Miner Res 25:948-959

13. Padhi D, Jang G, Stouch B, Fang L, Posvar E (2011) Single-dose, placebo-controlled, randomized study of AMG 785, a sclerostin monoclonal antibody. J Bone Miner Res 26:19-26

14. Li X, Zhang Y, Kang H, Liu W, Liu P, Zhang J, Harris SE, Wu D (2005) Sclerostin binds to LRP5/6 and antagonizes canonical Wnt signaling. J Biol Chem 280:19883-19887

15. Semenov M, Tamai K, He X (2005) SOST is a ligand for LRP5/ LRP6 and a Wnt signaling inhibitor. J Biol Chem 280:2677026775

16. Krause C, Korchynskyi O, de Rooij K, Weidauer SE, de Gorter DJ, van Bezooijen RL, Hatsell S, Economides AN, Mueller TD, Lowik CW, ten Dijke P (2010) Distinct modes of inhibition by sclerostin on bone morphogenetic protein and Wnt signaling pathways. J Biol Chem 285:41614-41626

17. Li X, Ominsky MS, Niu QT, Sun N, Daugherty B, D'Agostin D, Kurahara C, Gao Y, Cao J, Gong J, Asuncion F, Barrero M, Warmington K, Dwyer D, Stolina M, Morony S, Sarosi I, Kostenuik PJ, Lacey DL, Simonet WS, Ke HZ, Paszty C (2008)
Targeted deletion of the sclerostin gene in mice results in increased bone formation and bone strength. J Bone Miner Res 23:860-869

18. Balemans W, Ebeling M, Patel N, Van Hul E, Olson P, Dioszegi M, Lacza C, Wuyts W, Van Den Ende J, Willems P, Paes-Alves AF, Hill S, Bueno M, Ramos FJ, Tacconi P, Dikkers FG, Stratakis C, Lindpaintner K, Vickery B, Foernzler D, Van Hul W (2001) Increased bone density in sclerosteosis is due to the deficiency of a novel secreted protein (SOST). Hum Mol Genet 10:537-543

19. Brunkow ME, Gardner JC, Van Ness J, Paeper BW, Kovacevich BR, Proll S, Skonier JE, Zhao L, Sabo PJ, Fu Y, Alisch RS, Gillett L, Colbert T, Tacconi P, Galas D, Hamersma H, Beighton P, Mulligan J (2001) Bone dysplasia sclerosteosis results from loss of the SOST gene product, a novel cystine knot-containing protein. Am J Hum Genet 68:577-589

20. Balemans W, Patel N, Ebeling M, Van Hul E, Wuyts W, Lacza C, Dioszegi M, Dikkers FG, Hildering P, Willems PJ, Verheij JB, Lindpaintner K, Vickery B, Foernzler D, Van Hul W (2002) Identification of a $52 \mathrm{~kb}$ deletion downstream of the SOST gene in patients with van Buchem disease. J Med Genet 39:91-97

21. Staehling-Hampton K, Proll S, Paeper BW, Zhao L, Charmley P, Brown A, Gardner JC, Galas D, Schatzman RC, Beighton P, Papapoulos S, Hamersma H, Brunkow ME (2002) A 52-kb deletion in the SOST-MEOX1 intergenic region on 17q12-q21 is associated with van Buchem disease in the Dutch population. Am J Med Genet 110:144-152

22. Ralston SH, Uitterlinden AG (2010) Genetics of osteoporosis. Endocr Rev 31:629-662

23. Power J, Poole KE, van Bezooijen R, Doube M, Caballero-Alias AM, Lowik C, Papapoulos S, Reeve J, Loveridge N (2010) Sclerostin and the regulation of bone formation: effects in hip osteoarthritis and femoral neck fracture. J Bone Miner Res 25:1867-1876

24. De Souza RL, Matsuura M, Eckstein F, Rawlinson SC, Lanyon LE, Pitsillides AA (2005) Non-invasive axial loading of mouse tibiae increases cortical bone formation and modifies trabecular organization: a new model to study cortical and cancellous compartments in a single loaded element. Bone 37:810-818

25. Sugiyama T, Price JS, Lanyon LE (2010) Functional adaptation to mechanical loading in both cortical and cancellous bone is controlled locally and is confined to the loaded bones. Bone 46:314-321

26. Sugiyama T, Galea GL, Lanyon LE, Price JS (2010) Mechanical loading-related bone gain is enhanced by tamoxifen but unaffected by fulvestrant in female mice. Endocrinology 151:5582-5590

27. Srinivasan S, Weimer DA, Agans SC, Bain SD, Gross TS (2002) Low-magnitude mechanical loading becomes osteogenic when rest is inserted between each load cycle. J Bone Miner Res 17:1613-1620

28. McKenzie JA, Silva MJ (2011) Comparing histological, vascular and molecular responses associated with woven and lamellar bone formation induced by mechanical loading in the rat ulna. Bone 48:250-258

29. Prasad J, Wiater BP, Nork SE, Bain SD, Gross TS (2010) Characterizing gait induced normal strains in a murine tibia cortical bone defect model. J Biomech 43:2765-2770

30. Stadelmann VA, Hocke J, Verhelle J, Forster V, Merlini F, Terrier A, Pioletti DP (2009) 3D strain map of axially loaded mouse tibia: a numerical analysis validated by experimental measurements. Comput Methods Biomech Biomed Engin 12:95-100

31. Lynch ME, Main RP, Xu Q, Walsh DJ, Schaffler MB, Wright TM, van der Meulen MCH (2010) Cancellous bone adaptation to tibial compression is not sex-dependent in growing mice. J Appl Physiol 109:685-691

32. Vincent C, Findlay DM, Welldon KJ, Wijenayaka AR, Zheng TS, Haynes DR, Fazzalari NL, Evdokiou A, Atkins GJ (2009) Proinflammatory cytokines TNF-related weak inducer of apoptosis (TWEAK) and TNFalpha induce the mitogen-activated protein 
kinase (MAPK)-dependent expression of sclerostin in human osteoblasts. J Bone Miner Res 24:1434-1449

33. Heiland GR, Zwerina K, Baum W, Kireva T, Distler JH, Grisanti M, Asuncion F, Li X, Ominsky M, Richards W, Schett G, Zwerina J (2010) Neutralisation of Dkk-1 protects from systemic bone loss during inflammation and reduces sclerostin expression. Ann Rheum Dis 69:2152-2159

34. Mosley JR, March BM, Lynch J, Lanyon LE (1997) Strain magnitude related changes in whole bone architecture in growing rats. Bone 20:191-198

35. Gross TS, Edwards JL, McLeod KJ, Rubin CT (1997) Strain gradients correlate with sites of periosteal bone formation. J Bone Miner Res 12:982-988

36. Nicolella DP, Bonewald LF, Moravits DE, Lankford J (2005) Measurement of microstructural strain in cortical bone. Eur $\mathrm{J}$ Morphol 42:23-29

37. Nicolella DP, Moravits DE, Gale AM, Bonewald LF, Lankford J (2006) Osteocyte lacunae tissue strain in cortical bone. J Biomech 39:1735-1743

38. Silvestrini G, Ballanti P, Sebastiani M, Leopizzi M, Di Vito M, Bonucci E (2008) OPG and RANKL mRNA and protein expressions in the primary and secondary metaphyseal trabecular bone of PTH-treated rats are independent of that of SOST. J Mol Histol 39:237-242

39. Yamane H, Sakai A, Mori T, Tanaka S, Moridera K, Nakamura T (2009) The anabolic action of intermittent PTH in combination with cathepsin $\mathrm{K}$ inhibitor or alendronate differs depending on the remodeling status in bone in ovariectomized mice. Bone 44:10551062

40. Gross TS, Rubin CT (1995) Uniformity of resorptive bone loss induced by disuse. J Orthop Res 13:708-714

41. Gaudio A, Pennisi P, Bratengeier C, Torrisi V, Lindner B, Mangiafico RA, Pulvirenti I, Hawa G, Tringali G, Fiore CE (2010) Increased sclerostin serum levels associated with bone formation and resorption markers in patients with immobilization-induced bone loss. J Clin Endocrinol Metab 95:2248-2253

42. Mirza FS, Padhi ID, Raisz LG, Lorenzo JA (2010) Serum sclerostin levels negatively correlate with parathyroid hormone levels and free estrogen index in postmenopausal women. J Clin Endocrinol Metab 95:1991-1997

43. Kramer I, Loots GG, Studer A, Keller H, Kneissel M (2010) Parathyroid hormone (PTH)-induced bone gain is blunted in SOST overexpressing and deficient mice. J Bone Miner Res 25:178-189

44. Drake MT, Srinivasan B, Modder UI, Peterson JM, McCready LK, Riggs BL, Dwyer D, Stolina M, Kostenuik P, Khosla S (2010) Effects of parathyroid hormone treatment on circulating sclerostin levels in postmenopausal women. J Clin Endocrinol Metab 95:5056-5062

45. Sugiyama T, Saxon LK, Zaman G, Moustafa A, Sunters A, Price JS, Lanyon LE (2008) Mechanical loading enhances the anabolic effects of intermittent parathyroid hormone (1-34) on trabecular and cortical bone in mice. Bone 43:238-248 